Results Out of the total 743 workers, majority were males (71\%). Higher proportion of workers $(59 \%)$ belonged to 20 39 year age group. Twenty four percent and $28 \%$ of the study population smoked cigarettes and drank alcohol regularly respectively. Only $6 \%$ of workers had a pre employment medical examination and periodic medical examinations. Of the workers only $38 \%$ were using personal protective equipment. $47 \%$ of workers reported that compensation claims were paid for accidents at the factory. $62 \%$ of the workers knew how to use a first aid box.

Conclusion The provision of occupational health services to workers in small scale industries is not satisfactory and needs improvement.

\section{PERCEPTIONS AND AWARENESS OF RISK IN THE WORKPLACE AMONG MIGRANTS TO AUSTRALIA}

${ }^{1}$ A Reid, ${ }^{1}$ Santos, ${ }^{2}$ Hard, ${ }^{1}$ Fritschi, ${ }^{3}$ Lenguerrand, ${ }^{4}$ LaMontagne. ${ }^{1}$ The University of Western Australia, Crawley, Australia; ${ }^{2}$ Medical Research Council, Social and Public Health Sciences Unit, Glasgow, United Kingdom; ${ }^{3}$ The University of Bristol, Bristol, United Kingdom; ${ }^{4}$ The University of Melbourne, Melbourne, Australia

\subsection{6/oemed-2013-101717.37}

Objectives Migration has been important to the wealth and development of Australia and one in four Australians are born abroad. Our analysis of the national Multi-Purpose Household Survey showed generally higher risks in specific industrial settings but not among migrant workers. Anecdotal evidence, however, suggest that migrant workers are exposed to more workplace hazards. In qualitative interviews we explored perceptions of health and safety and experiences in home countries and in Australia.

Methods 92 purposively sampled migrant workers took part in individual interviews $(n=15)$ or 8 focus groups $(n=77)$ from 22 countries (20 from low to middle income countries (LMICs)). Migrants were sampled via migrant organisations, trade unions or via advertisements in the local press. Interviews were digitally recorded, anonymised, and transcribed verbatim. NVivo 10 was used to organise and explore coded transcripts. A concurrent thematic analysis was conducted. Themes around each category were verified and confirmed by constant comparison and searching across all interviews for similar themes and categories for analysis.

Results The key themes that emerged included poor understanding of occupational health and safety, particularly among those from LMICs and even among those with a relatively higher education level; general perception that Australia was a safe place to work in although there were reports of risky practices (e.g. long working hours) and overt discriminatory practices such as racism or bullying in workplaces; and a general resilience towards adversity in workplaces. Social support networks (e.g. via churches) or the commitment to sending remittances to family in home countries played an important role in coping with such adversity. Many tried to explain the racism as humour, and incidents were rarely reported.

Conclusion These findings of a poor understanding of health and safety among migrants from LMICs, shed some light on the context which might result in under-reporting in national surveys.

\section{OCCUPATIONAL HAZARDS IN SOME SELECTED SMALL SCALE INDUSTRIES IN A DISTRICT OF SRI LANKA}

${ }^{1} \mathrm{~S}$ A I K Suraweera, ${ }^{2}$ Senanayake, ${ }^{2}$ Wijesinghe. ${ }^{1}$ Environmental and Occupational Health Unit, Colombo, Sri Lanka; ${ }^{2}$ Ministry of Health, Colombo, Sri Lanka

\subsection{6/oemed-2013-101717.38}

Occupational hazards in some selected small scale industries in a district of Sri Lanka

Objective To identify occupational hazards in the work environment in small scale industiries in a district of Sri Lanka

Method A descriptive cross sectional study was carried out in selected categories of small scale industries namely food and beverages, apparel, non metallic mineral products and fabricated metal products in a district of Sri Lanka. A small scale industry was defined as a work setting with less than 20 workers. A sample of 102 factories was randomly selected using Census of Industries Sri Lanka in 2003/2004. Interviewer administered pre tested checklist was used for data collection.

Results Out of $102,78.4 \%(\mathrm{n}=80)$ of the factories did not have safety signs displayed while machines were properly guarded only in $25(24.5 \%)$ of the factories. The working environment was found to be accident prone in $38.2 \%(n=39)$ of the industries. Lighting was adequate in $93(91.2 \%)$ and noise was found to be excessive in 35 (34.3\%) factories.

A functioning safety committee was available in 5 (4.9\%) industries while workers trained in occupational safety and first aid, were present only in $18.6 \%(n=19)$ and $23.5 \%(n=24)$ of the factories respectively. Only $24(23.5 \%)$ industries had a protocol developed to act in an emergency situation

Accidents were recorded only in $16(15.7 \%)$ factories. Separate meal room and a changing room were available in $62.7 \%$ ( $\mathrm{n}$ $=64$ ) of the factories.

Conclusion The working environment of small scale industries was found to be unsatisfactory. Detailed studies are warranted to assess the hazards in depth. However, measures should be planned to improve the working environment and health of small scale industry workers since they represent a significant percent of the labour force in Sri Lanka.

\section{PREVALENCE OF SELECTED HEALTH ISSUES AMONG WORKERS IN SMALL SCALE INDUSTRIES IN A DISTRICT IN SRI LANKA}

${ }^{1} \mathrm{~S}$ A I K Suraweera, ${ }^{2}$ Senanayake, ${ }^{2}$ Wijesinghe. ${ }^{1}$ Environmental and Occupational Health Unit, Colombo, Sri Lanka; ${ }^{2}$ Ministry of Health, Colombo, Sri Lanka

\subsection{6/oemed-2013-101717.39}

Prevalence of selected health issues among workers in small scale industries in a district in Sri Lanka

Objectives To assess the prevalence of selected health issues among workers, in small scale industries in a district in Sri Lanka

Method A cross sectional study was conducted among workers in four selected small scale industry categories namely food and beverages, apparel, non metallic mineral products and fabricated metal products in a district of Sri Lanka. A small scale industry was defined as a work setting with less than 20 workers. The census of Industries Sri Lanka 2003/2004 was used as the sampling frame. Cluster sampling using probability proportionate to size of the workers was done. Cluster size was eight and eighty clusters were selected. Data was collected using a pre-tested interviewer administered questionnaire.

Results Analysis was based on 727 workers. A majority (63.8\%, $\mathrm{n}=464)$ were in the age group 20-39 years and $499(68.6 \%)$ 